\title{
Outcomes of segmental tibia fractures treated with circular external fixation at a single centre in a developing world setting
}

\author{
$\mathrm{O}^{\prime}$ Connor $\mathrm{M}^{1}$, Marais $\mathrm{LC}^{2}$, Ferreira $\mathrm{N}^{3}$ \\ ${ }^{1}$ MBBCh; Orthopaedic registrar, Department of Orthopaedic Surgery, Grey's Hospital, Nelson R Mandela School of Clinical Medicine, University of KwaZulu-Natal, \\ South Africa \\ 2 MBChB, FC Orth(SA), MMed(Orth), PhD; Associate Professor and Head of Department of Orthopaedic Surgery, Nelson R Mandela School of Clinical Medicine, \\ University of KwaZulu-Natal, South Africa \\ ${ }_{3}^{3}$ BSc, MBChB, FC Orth(SA), MMed(Orth), PhD; Associate Professor and Head of Clinical Unit: Tumour, Sepsis and Reconstruction, Division of Orthopaedic Surgery, \\ Faculty of Medicine and Health Sciences, Stellenbosch University, Cape Town, South Africa
}

Corresponding author: Dr Megan O'Connor, Department of Orthopaedic Surgery, Grey's Hospital, Nelson R Mandela School of Clinical Medicine, University of KwaZulu-Natal, Pietermaritzburg, South Africa; tel: +27 338973000 ; email: megsmohican@gmail.com

\begin{abstract}
Background: Segmental tibia fractures historically are treated with special consideration due to their complexity, high complication rate and the propensity for associated injuries. Circular external fixators (CEFs) offer several potential advantages in this situation due to their modularity and minimally invasive application. These advantages have been demonstrated in several developed countries but not as yet in the developing world. This analysis aimed to evaluate if these beneficial outcomes are reproducible in a developing world environment.
\end{abstract}

Methods: We retrospectively assessed the data pertaining to skeletally mature patients who sustained segmental tibia fractures and were treated with CEFs between January 2008 to December 2015. These cases were performed in our tertiary hospital and overseen by a consultant with limb reconstruction experience. Union rates, time to union and complications encountered over a median of 10 months (range 6-20 months) follow-up period were among the outcomes measured.

Results: Fourteen male and three female patients with a median age of 34 years were included. Sixteen patients (94\%) were injured as a result of high energy trauma, nine patients (53\%) had associated injuries, fourteen cases (83\%) were compound injuries and there were no cases of compartment syndrome. Fifteen patients (88\%) united with the use of the initial CEF at a median of 25 weeks (16-52 weeks), the two cases of non-union were treated with adjustments to the CEF and distraction across the affected fracture site with ultimate union. Two cases (12\%) of superficial pin infection were encountered and one case (6\%) of deep infection occurred after union of the fractures necessitating removal of the CEF. Three cases (18\%) developed malunion.

Conclusion: Circular external fixators used for segmental tibia fractures, when applied in a developing setting by clinicians with limb reconstruction experience, display a satisfactory union rate and acceptable complication frequency, as compared to that achieved in developed countries.

Level of evidence: Level 4

Key words: tibia, segmental fracture, circular fixator, llizarov, non-union, infection

Citation: O'Connor M, Marais LC, Ferreira N. Outcomes of segmental tibia fractures treated with circular external fixation at a single centre in a developing world setting. SA Orthop J 2018;17(3):41-46. http://dx.doi.org/10.17159/2309-8309/2018/v17n3a5

Editor: Prof S Maqungo, University of Cape Town

Received: November 2017

Accepted: May 2018

Published: August 2018

Copyright: (c) 2018 O'Connor M. This is an open-access article distributed under the terms of the Creative Commons Attribution Licence, which permits unrestricted use, distribution and reproduction in any medium, provided the original author and source are credited.

Funding: The study was retrospective in nature and as such required no funding.

Conflict of interest: The authors report no conflicts of interest with respect to this study. 


\section{Introduction}

Segmental tibia fractures are a separate entity from isolated diaphyseal tibia fractures due to their intrinsic injury characteristics, management complexity and higher complication rates. ${ }^{1-4}$ Choice of fixation device requires special consideration in order to address these difficulties. A number of management options have been investigated with varying outcomes but collectively they report prolonged healing times, multiple operations and high complication rates. ${ }^{1,2,4-9}$

Circular external fixators (CEFs) have gained popularity for use with complex tibia fractures. They are currently recommended for use in high energy tibial trauma, periarticular tibial fractures, in the presence of infection, for open injuries and in deformity correction. ${ }^{10-12}$ This is due in part to its advantageous design in terms of modularity allowing application suited to a particular fracture pattern within the constraints of soft tissue injury. ${ }^{10,13}$ Its inherent biomechanical properties also see it termed in literature as 'true biologic fixation' that has been confirmed by reports indicating high union rates. ${ }^{1,13,14}$ These benefits make CEF an attractive option for the management of segmental tibia fractures.

These benefits have been demonstrated in some studies conducted in developed countries. These concluded that CEF is a reasonable method of treatment, with good union rates and few complications. No studies assessing this practice have been conducted in the developing world. ${ }^{1,14,15}$

The objective of this study was thus to assess if the outcomes of CEF for segmental tibia fractures obtained in the developed world are reproducible in a developing country.

\section{Patients and methods}

Consecutive patients who presented with segmental tibia fractures between January 2008 and January 2015, and who were treated with circular external fixation at our training hospital with limb reconstruction experience, were eligible for evaluation. Institutional ethics committee approval was obtained for this study. Patients were identified from a prospectively collected database. Inclusion criteria were patients with segmental tibia fractures who completed treatment with definitive CEF. Patients were only excluded if they were not skeletally mature.

Patients were categorised according to: demographics (including smoking history, human immunodeficiency virus [HIV] status and presence of diabetes), fracture classification, compound grading if open (Gustilo and Anderson), ${ }^{16}$ mechanism of injury and presence of associated injuries. All patients were offered voluntary counselling and testing for HIV during their hospital stay.

Segmental fractures were defined as fractures where at least two distinct fracture lines were separated by a cylindrical intermediate segment; the Orthopaedic Trauma Association (OTA) classify this as OTA 42-C2. A further classification, as suggested by Melis et al., was used to define the levels of the individual fracture lines (Table I). ${ }^{6}$

Table I: The Melis classification of segmental tibia fractures ${ }^{6}$

Type I The fracture lines are situated proximally, so that the proximal fracture lies in the upper third of the shaft and the distal fracture lies in the middle third.

Type II The fracture lines are situated distally, so that the proximal fracture lies in the middle third of the shaft and the distal fracture lies in the lower third.

Type III The fracture lines are at the extremes of the shaft and there is a long intermediate fragment.

Type IV The fracture lines are close to one another and there is a short intermediate fragment in the middle third of the shaft.
Fourteen patients (82\%) with open fractures were managed according to a standardised treatment protocol that included prophylactic antibiotics on arrival in the emergency department and urgent surgical debridement followed by temporary fracture stabilisation with a mono-lateral external fixator. A 48-hour wound inspection and closure was performed by either delayed primary closure in nine cases (64\%) or soft tissue flap in two cases (14\%). The remaining three cases (18\%) had delayed closure more than 48 hours post initial debridement.

CEF included constructs composed of rings and fine wire fixation only, as well as hexapod fixators with adjustable struts. CEF were constructed and applied according to the specific fracture pattern. All CEFs were applied under the supervision of a consultant with limb reconstruction experience. Application of the CEF was performed as outlined in the Instructional Article of Ferreira et al. ${ }^{17}$ A proximal reference wire was placed parallel to the knee joint, followed by frame application. Thereafter the distal reference wire was placed parallel to the ankle joint. This achieved coronal plane alignment. Sagittal plane alignment was completed under fluoroscopy and all wires were tensioned to $130 \mathrm{~kg}$. The proximal and distal bone segments were stabilised with ring blocks while the intercalary segment was stabilised with a single ring (Figure 1). Fixation was concluded with the application of at least two tensioned wires per ring. Hexapod external fixators were used in cases where acute fracture reduction was unsuccessful. These CEFs were applied using the 'rings first' method $^{18}$ (Figure 2). This

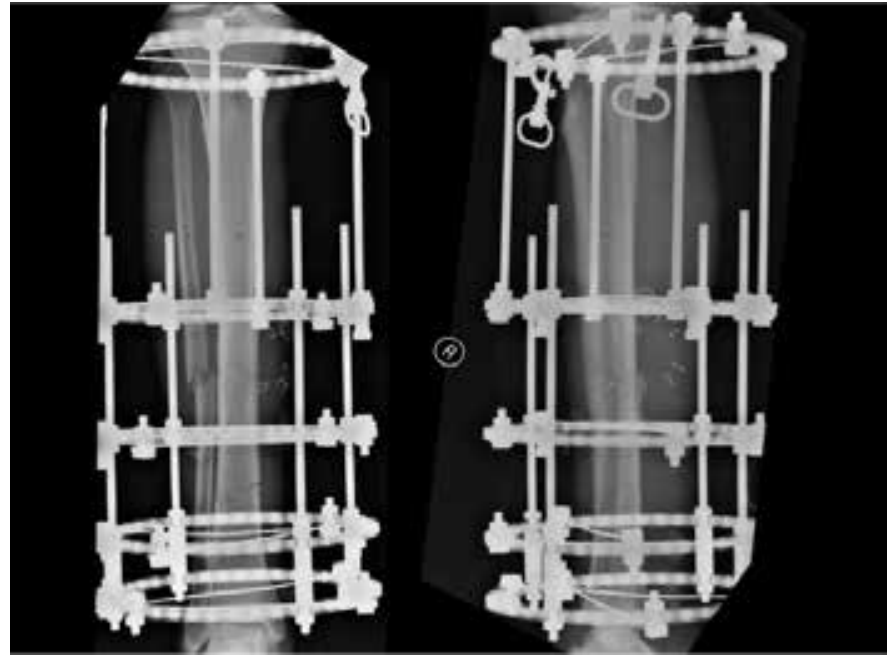

Figure 1. Antero-posterior and lateral radiographs depicting the construct used to stabilise the segmental fracture of a Melis type II fracture

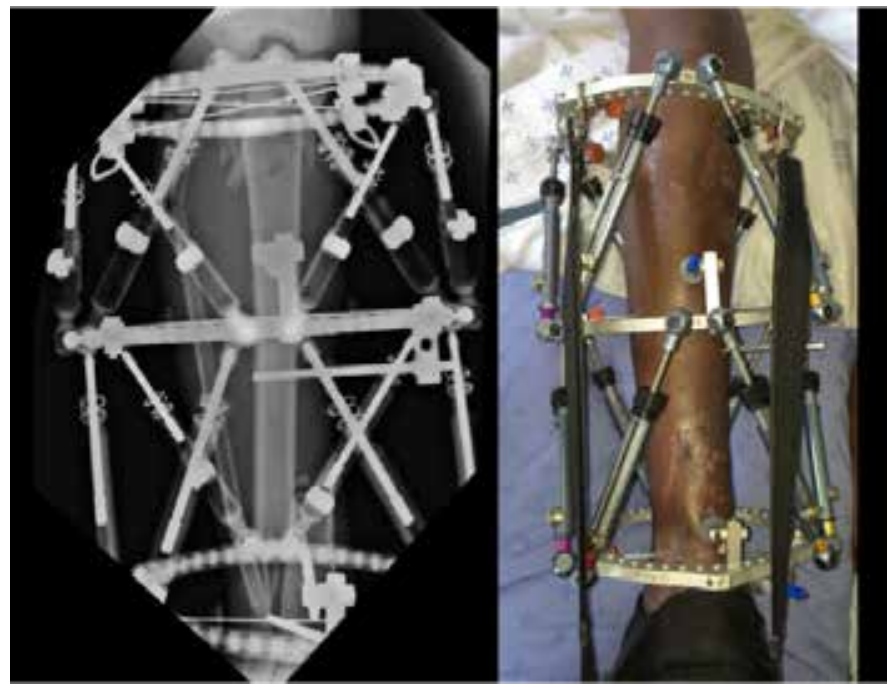

Figure 2. Stacked TL-Hex for Melis type III fracture of the right tibia 
Table II: Checketts and Otterburn classification of pin-track infection ${ }^{20}$

\begin{tabular}{|c|c|c|}
\hline Grade & Characteristics & Treatment \\
\hline \multicolumn{3}{|c|}{ Minor infection } \\
\hline 1 & Slight redness and little discharge & Improved pin-site care \\
\hline 2 & Redness of the skin, discharge, pain and tenderness in the soft tissue & Improved pin-site care and oral antibiotics \\
\hline 3 & Grade 2 but no improvement with oral antibiotics & $\begin{array}{l}\text { Affected pin or pins resited and external fixation } \\
\text { can be continued }\end{array}$ \\
\hline \multicolumn{3}{|c|}{ Major infection } \\
\hline 4 & $\begin{array}{l}\text { Severe soft tissue infection involving several pins, sometimes with associated } \\
\text { loosening of the pin }\end{array}$ & External fixation must be abandoned \\
\hline 5 & Grade 4 but radiographic changes & External fixation must be abandoned \\
\hline 6 & $\begin{array}{l}\text { Infection after fixator removal. Pin track heals initially, but will subsequently } \\
\text { break down and discharge in intervals. Radiographs show new bone formation } \\
\text { and sometimes sequestra }\end{array}$ & Curettage of the pin tract \\
\hline
\end{tabular}

method entailed the independent, orthogonal application of one ring to each bone segment. Thereafter a total of 12 struts were applied, six variable length struts were attached between each set of rings in a stacked fashion. Post-operative radiographs were used to input variables to provide a programme for gradual deformity correction at a rate of $1 \mathrm{~mm}$ per day for each set of struts. This programme was commenced once the soft tissues were deemed suitable.

All patients were rehabilitated post-operatively with physiotherapists emphasising adjacent joint range of motion, normalisation of gait pattern and full weight bearing on the affected limb. Early weight bearing was promoted, and patients were encouraged to mobilise full weight bearing as soon as pain permitted. The patients were initiated on a pin-site care protocol entailing daily cleaning with chlorhexidine. ${ }^{19}$ Outpatient follow-up was scheduled at two-weekly intervals until a robust rehabilitation programme was established. Thereafter, the interval between follow-up appointments was increased to four weeks.

Complications were recorded for patients. Pin-site infection was graded according to the Checketts and Otterburn classification ${ }^{20}$ (Table II).

Removal of the frame was considered once radiographic healing of three cortices was evident. At this point the fixator was dynamised and the site of the uniting fracture manually stressed to determine clinical union. If the patient was able to walk without pain, they were allowed to return home with a fully dynamised frame and encouraged to mobilise, fully weight bearing, for a period of two weeks as a 'trial of union'. Repeat radiographs at follow-up were compared with those before the trial; if no deformity had developed, union was deemed confirmed and the external fixator removed. No additional protection was provided in the form of a cast or boot after removal of the frame. Time to union (TTU) was thus defined as the duration for which the patient had the fixator applied. The TTU of the two patients with nonunion, who underwent conversion to hexapod fixators, was used in calculating the median TTU.

Malunion was defined as a coronal deformity of more than $5^{\circ}$ or a sagittal deformity of greater than $10^{\circ} .{ }^{21}$ Non-union was classified according to several criteria including: atrophic or hypertrophic, stiff or mobile, with or without deformity, with or without bone loss, and septic or not.22

\section{Results}

Seventeen patients met the inclusion criteria (Table III). No patients were excluded. The final study cohort consisted of
14 men and three women with a median age of 34 years (range 19-73; interquartile range [IQR] 17). Median follow-up was 10 months (range 6-20 months; IQR 4).

Open fractures were observed in 14 patients (82\%). Of these, seven fractures (50\%) were classified as Gustilo-Anderson IIIA and seven (50\%) as Gustilo-Anderson IIIB. The remaining three patients $(18 \%)$ had closed fractures. According to the Melis classification, four injuries (24\%) were found to be Melis I, six (35\%) Melis II, four (24\%) Melis III and three (18\%) Melis IV. The mechanism of injury for five patients (29\%) was a motor vehicle accident (MVA), for 11 patients (66\%) was a pedestrian vehicle accident (PVA) and one patient (6\%) was assaulted. Nine patients (53\%) presented with multiple injuries. These associated injuries included contralateral tibia fractures in three patients (18\%), femur fractures in three patients (18\%), cervical spine injuries in two patients (12\%), pelvic fractures in two patients $(12 \%)$, blunt chest trauma in one patient $(6 \%)$, head injuries in two patients (12\%) and blunt abdominal trauma in one patient (6\%).

HIV infection was identified in two patients $(12 \%)$ with cluster of differentiation $4\left(\mathrm{CD}^{+}\right)$counts of 300 and 450 cells $/ \mathrm{mm}^{3}$ respectively. Both patients were on highly active anti-retroviral (HAART) treatment. Four patients (24\%) were active smokers. One patient (6\%) was diabetic.

Thirteen injuries (77\%) were treated in traditional Ilizarovtype fine wire CEFs. These included seven (41\%) TrueLok fixators (Orthofix, Verona, Italy) and six (35\%) Ilizarov fixators (Smith \& Nephew, Memphis, Tennessee). Hexapod external fixation was used in four patients (24\%) and consisted of three (18\%) Taylor Spatial Frames (Smith \& Nephew, Memphis, Tennessee) and one (6\%) TrueLok-Hex fixators (Orthofix, Verona, Italy). The median duration of external fixator treatment was 25 weeks (range 16-52 weeks; IQR 17).

Union was achieved at both fracture sites after initial management in 15 out of 17 cases (88\%). The two cases (12\%) that failed to unite initially both had non-unions of the distal fracture site. These injuries were classified as Melis III and Melis IV respectively. Both were classified as hypertrophic non-unions and successfully treated with closed distraction after conversion to a hexapod construct. There were three cases (18\%) of malunion, two Melis type III cases (12\%) developed valgus deformities of the proximal fracture site of $6^{\circ}$ and $8^{\circ}$ respectively. The remaining case (6\%), a Melis type I fracture pattern, had a $22^{\circ}$ procurvatum deformity of the distal fracture.

Pin-site infection was a common complication and occurred in three patients (18\%) patients. According to the Checketts and Otterburn classification two (12\%) were minor cases and responded well to local pin-site care and oral antibiotics. One 
Table III: Patient demographics

\begin{tabular}{|c|c|c|c|c|c|c|c|}
\hline \multicolumn{8}{|c|}{ Total patients $=17$} \\
\hline Male & 14 & Female & 3 & & & & \\
\hline Median age & $34^{1}$ & & & & & & \\
\hline HIV ${ }^{2}$ positive & 2 & & & & & & \\
\hline Diabetic & 1 & & & & & & \\
\hline Smoker & 4 & & & & & & \\
\hline Right & 11 & Left & 6 & & & & \\
\hline Open & 14 & Closed & 3 & & & & \\
\hline $\mathrm{GA}^{3} \| \mathrm{Ila}$ & 7 & GA $\| \mathrm{b}$ & 7 & & & & \\
\hline Melis I & 4 & Melis II & 6 & Melis III & 4 & Melis IV & 3 \\
\hline Polytrauma & 9 & & & & & & \\
\hline Tibia & 3 & Femur & 3 & C-spine & 2 & Abdomen & 1 \\
\hline Pelvis & 2 & Chest & 1 & Head & 2 & & \\
\hline $\mathrm{MVA}^{4}$ & 5 & PVA $^{5}$ & 11 & Assault & 1 & & \\
\hline TrueLok & 7 & Ilizarov & 6 & TL-Hex 6 & 1 & $\mathrm{TSF}^{7}$ & 3 \\
\hline Pin infection & 3 & & & & & & \\
\hline$C \& O^{8} 2$ & 2 & C\&O 5 & 1 & & & & \\
\hline Time to union & $25^{9}$ & $\mathrm{IQR}^{10}$ & 17 & & & & \\
\hline Melis I & 42.5 & Melis II & 18.5 & Melis III & 26.5 & Melis III & 31 \\
\hline Follow-up & $10^{11}$ & IQR & 4 & & & & \\
\hline Malunion & 3 & & & & & & \\
\hline Non-union & 2 & & & & & & \\
\hline
\end{tabular}

${ }^{7}$ Age in years, ${ }^{2}$ Human immunodeficiency virus, ${ }^{3}$ Gustilo and Anderson grade, ${ }^{4}$ Motor vehicle accident, ${ }^{5}$ Pedestrian vehicle accident, ${ }^{6}$ TrueLok-Hex,

${ }^{7}$ Taylor Spatial Frame, ${ }^{8}$ Checketts and Otterburn grade, ${ }^{9}$ Time in weeks, ${ }^{10}$ Interquartile range, ${ }^{11}$ Time in months

patient (6\%) developed a Checketts and Otterburn grade 5 pin-site infection after the fracture was deemed united, in the 'trial period' before removal; this necessitated removal of the external fixator.

The median TTU was 25 weeks (range 16-52; IQR 17). The TTU was variable across those differentiated according to the Melis classification. Melis type I median TTU was 42.5 weeks (range 25-52; IQR 16.5), Melis II was 18.5 weeks (range 16-25; IQR 5.5), Melis III was 26.5 weeks (range 18-45; IQR 10.5) and Melis IV (range 25-37; IQR 9.5) was 31 weeks. The small sample size prohibited more robust statistical analysis of the data.

\section{Discussion}

Segmental fractures of the tibia are complex injuries with an incidence of between $3 \%$ and $13 \%$. 2,3,5,9,10,15,23-25 Approximately $80 \%$ of cases occur in males at an average age of 41.1 years. Our data reflects a similar male preponderance of 14 patients (82\%); however, our collection of patients had a lower median age of 34 years.

Previous studies show that these fractures result from high energy trauma and our data was representative of this. On average $74 \%$ of cases in the literature are as a result of MVAs; in our data set, five injuries (29\%) were secondary to MVA but an additional 11 injuries (65\%) resulted from PVA. 1,14,15,23-25 In association with the high energy mechanism an increased incidence of multiple injuries and open fractures was also seen. Some previous reports noted that all patients with segmental fractures sustained multiple injuries, but on average $58 \%(29 \%-100 \%)$ of cases have associated injuries and $64 \%(35 \%-100 \%)$ of fractures are compound in nature. ${ }^{1,2,5,6,8,14,15,23-25}$ Our data is in keeping with these values where ten patients (60\%) had associated injuries and 14 fractures (82\%) were compound. There is also a concomitant risk of developing compartment syndrome quoted as high as $50 \%$ in one study; our data did not reflect this as no compartment syndrome was encountered. ${ }^{23,25}$ Perhaps this finding is due in part to the high proportion of open injuries; it could also reflect the minimally invasive application of the construct and thus minimisation of further soft tissue injury and swelling.

A previous investigation of segmental fractures performed by Ozturkmen et al. classified their 24 patients according to the Melis classification with a comparable distribution of patients in each group. Melis I seven patients (29\%) to our four patients (24\%), Melis II nine patients (38\%) to our six patients (35\%), Melis III five patients (21\%) to our four patients (24\%) and Melis IV three patients $(13 \%)$ in their study and three patients $(18 \%)$ in ours. ${ }^{1}$ 
Treatment of these injuries historically has been complex, and no single fixation method is considered gold standard. Intramedullary nailing (IMN) and plating are the most commonly proposed options other than external fixation. Plating has a high rate of wound complications and compartment syndrome, and further devascularises the bone. ${ }^{1,10} \mathrm{~A}$ study by Rommens et al. highlighted these problems reporting a $60 \%$ complication rate, including a $25 \%$ occurrence of infection and wound complications. ${ }^{4}$

The controversy with regard to IMN fixation is whether one should ream or not. Reaming further devascularises the endosteum and increases the infection rate in compound and high energy injuries; it is also difficult to control the intercalary fragment while reaming in segmental fractures. ${ }^{1,5,10,15}$ Choosing not to ream is not without consequences; studies including unreamed nails reported high deep infection rates as well. ${ }^{7}$ Irrespective of reaming or not, some studies report that IMN used for segmental injuries has high malunion rates and high re-operation rates. A potential bias is also present with the reporting of union with IMN as there is no clinical test for the strength of union while the implant remains in situ. ${ }^{7,10,15}$ Despite this information, two review articles have suggested the treatment of choice for closed segmental injuries be reamed, locked IMN. ${ }^{25}$ The review by Mcmurtry et al. ${ }^{24}$ further recommends unreamed, locked IMN for open segmental fractures. McMahon et al. ${ }^{25}$ do suggest, however, that in open segmental injuries CEF may provide the best results.

The potential advantages of CEF have meant that it has gained popularity for the management of complex tibia fractures. The apparatus is minimally invasive leaving a 'small footprint', thus making it suitable to use in injuries with significant soft tissue compromise where flaps may be necessary and further devascularisation of tissue should be avoided. $13,15,17,23,24,26$ Furthermore, it can be tailored to the specific fracture pattern due to its modularity. ${ }^{15,17}$ Its specific biomechanics allow micromotion but prevent rotational and shear forces, resulting in the ideal environment for bone formation. ${ }^{13,15,17,27}$ The stability of the structure allows immediate weight bearing on the injured limb, decreasing the incidence of deep vein thrombosis. $10,13,15,24,26,28$ The apparatus is easily adjustable without the need for further surgery. In this manner the fracture site can be compressed or distracted, activating angiogenesis in an attempt to promote union. $1,10,13,15,24,26,29$

The disadvantages of circular fixators have been high rates of pin-site infection (up to 54\%) and poor patient tolerance. . $^{17,19}$ No cases of deep infection were reported in the studies by Ozturkmen and Foster who used ring fixators for the management of segmental fractures. ${ }^{1,14}$ This is in stark contrast to the studies where intramedullary devices or plating techniques were used. Here, deep infection rates as high as $35 \%$ have been reported. $2,5-8,23$ We had three cases (18\%) of pin-site infection; two of these cases $(12 \%)$ had superficial sepsis that were treated with pin-site care and oral antibiotics resulting in resolution and allowing frame retention until union. The third patient with sepsis developed a deep infection after union had been achieved and so the use of the ring fixator was abandoned. This patient was a smoker, sustained an open injury and had multiple other injuries that potentially contributed to the presence of this complication.

Another disadvantage of CEFs specific to the management of segmental injuries is their complexity. Giotakis et al. discussed specifically that adjustments to CEFs were conducted in a dedicated limb reconstruction unit. ${ }^{15}$ Similarly the CEFs applied in this study were performed or supervised by a consultant with limb reconstruction experience. Although application follows the same basic principles as a standard CEF, a high level of competency is required to manage the stacked constructs as well as their programmes and as such readers are cautioned that the same results may not be reproducible.
Available statistics for segmental tibial injuries using various fixation methods report a range of TTU spanning 19 to 40 weeks with the use of a LISS plate, monolateral external fixation or nail respectively. ${ }^{5,23}$ Several studies also show delayed healing at the distal fracture site, with union achieved at an average of 34 weeks as opposed the average for the proximal fracture of 30 weeks. ${ }^{1,5,7,15}$ Union rates historically are spread over a wide range; some studies reflect a rate as low as $50 \%$ whereas others quote a rate of $96 \%$. Caution must be used when interpreting these values as in some instances this union is achieved after multiple operations and the use of varied methods of fixation. 2,4-8

Ozturkmen et al., Foster et al. and Giotakis et al. reported mean TTU with the use of circular fixators for segmental tibia fractures as 38 weeks, 27.9 weeks and 21.7 weeks, respectively.1,14,15 We observed a TTU with a median time of 25 weeks. These studies also reported union rates of $92 \%, 93 \%$ and $90 \%$ respectively, concluding favourable results achieved with the use of CEF. We similarly had a union rate of $88 \%$, with the two cases of non-union healing after distraction in hexapod external fixators.

Ozturkmen et al. looked specifically at TTUs with respect to the proximal and distal fractures healing at 36 weeks and 40 weeks respectively. This finding validates other literature documenting slower healing in distal fractures of segmental injuries. ${ }^{7}$ Our TTU comparing Melis classification was contrary to this literature, yet similar to the findings of Giotakis et al., who found no significant difference in the healing times of proximal and distal fractures. In our study, Melis II injuries united most rapidly at a median of 18.5 weeks and Melis I injuries healed the slowest at a median of 42.5 weeks. The numbers in our study do not allow for statistical analysis and it should be observed that there are several confounding factors that could account for this. All the Melis type I patients had several associated injuries and significant soft tissue injury as opposed to the type II patients where only one patient was multiply injured. This has previously been found to negatively impact TTU. ${ }^{30}$

Foster et al. compared TTUs of open and closed fractures with closed fractures healing faster at a mean of 25 weeks and open fractures uniting on average at 30 weeks. These are both greater overall times than our median of 25 weeks. Our data, unlike Foster et al. reflected longer TTU of closed fractures at a median of 37 weeks compared to the 25 weeks of the open injuries. This could again be explained by the fact that all the patients with closed fractures had associated injuries unlike those with open injuries.

Giotakis et al. also made the observation of the large range of time across which union occurred in their patients (12.8-31weeks) and they attributed this to the heterogenous nature of these fractures. ${ }^{15}$ We had a similar finding with a range of 16-52 weeks. In addition to the heterogeneity of the fractures, this could also reflect the large variation of associated injuries in these patients.

We had two cases (12\%) of non-union in this study, both of which involved the distal fracture site. This again is similar to that reported by Giotakis et al. of two patients $(10 \%)$ that also occurred at the distal fracture site. ${ }^{15}$ The first patient was 30 years of age, had a compound injury (GA IIIb), which was closed primarily at the 48-hour re-debridement, and was a non-smoker. He was involved in a PVA and was classified as a Melis III. The second patient was a 60-year-old smoker who had a compound (GA IIla) injury, also closed primarily at the 48-hour re-debridement. He had associated pelvic and cervical spine injuries. He was also involved in a PVA and was classified Melis IV. The advantage of this mode of fixation was highlighted in these two cases with ease of conversion to a hexapod construct through which distraction could be applied to the non-union site, stimulating callous formation and ultimately resulting in union. These two patients united at 29 and 25 weeks respectively. 
There were several limitations to the study. The incidence of segmental fractures is low and our representative population was limited. This affects the robustness of any statistical evaluation performed. The follow-up period is inadequate to assess long-term outcomes such as septic sequelae in open injuries. Finally, there was a senior surgeon present at each of these cases with expertise in the application of ring fixators; these outcomes are therefore not necessarily reproducible with an inexperienced surgeon.

\section{Conclusion}

CEFs used for segmental tibia fractures, when applied in a developing setting by clinicians with limb reconstruction experience, display a satisfactory union rate and acceptable complication frequency, as compared to that achieved in developed countries. It can be considered a useful addition to the armamentarium with which to manage these complex injuries.

\section{Ethics statement}

Institutional ethics committee approval was obtained for this study.

\section{References}

1. Ozturkmen $\mathrm{Y}$, Karamehmetoglu M, Karadeniz H, Azboy I, Caniklioglu M. Acute treatment of segmental tibial fractures with the Ilizarov method. Injury, 2009;40:321-26.

2. Teraa M, Blokhuis TJ, Tang L, Leenen LP. Segmental tibial fractures: an infrequent but demanding injury. Clin Orthop Relat Res. 2013;471:2790-96.

3. Motsitsi NS. Management of segmental tibial fractures. East and Central African Journal of Surgery, 2006;12:72-76.

4. Rommens PM, Coosemans W, Broos PL. The difficult healing of segmental fractures of the tibial shaft. Arch Orthop Trauma Surg. 1989;108:238-42.

5. Reynders P. Open acute segmental tibial fracture fixation using the less invasive stabilisation system (LISS): Study of 23 consecutive cases. Injury. 2009;40:449-54.

6. Melis GC, Sotgiu F, Lepori M, Guido P. Intramedullary nailing in segmental tibial fractures. J Bone Joint Surg Am. 1981;63:1310-18.

7. Giannoudis PV, Hinsche AF, Cohen A, Macdonald A, Matthews SJ, Smith RM. Segmental tibial fractures: an assessment of procedures in 27 cases. Injury. 2003;34:756-62.

8. Bonnevialle $\mathrm{P}$, Cariven $\mathrm{P}$, Bonnevialle N, Mansat P, Martinel V, Verhaeghe L, Mansat M. [Segmental tibia fractures: a critical retrospective analysis of 49 cases]. Rev Chir Orthop Reparatrice Appar Mot. 2003;89:423-32.

9. Sarmiento A, Latta LL. Functional treatment of closed segmental fractures of the tibia. Acta Chir Orthop Traumatol Cech. 2008;75:325-31.

10. Sidharthan S, Sujith A, Rathod A, Pathak R. Management of high energy tibial fractures using Ilizarov apparatus. The Internet Journal of Orthopaedic Surgery. 2005;3.

11. Esmaeilnejad Ganji SM, Bahrami M, Joukar F. Ilizarov versus AO external fixator for the treatment of tibia open fractures. Iran Red Crescent Med . 2011;13:868-72.

12. Hasankhani E, Payvandi MT, Birjandinejad A. The Iliazarov ring external fixator in complex open fractures of the tibia. European Journal of Trauma. 2006;32:63-68.

13. Fragomen AT, Rozbruch SR. The mechanics of external fixation. HSS J. 2007;3:13-29.

14. Foster PAL, Barton S, Jones SCE, Britten S. Treatment of segmental tibial fractures by the llizarov method: an update. Orthopaedic Proceedings. 2013;95-B.

15. Giotakis N, Panchani SK, Narayan B, Larkin JJ, Al Maskari S, Nayagam S. Segmental fractures of the tibia treated by circular external fixation. J Bone Joint Surg Br. 2010;92:687-92.

16. Gustilo RB, Anderson JT. Prevention of infection in the treatment of one thousand and twenty-five open fractures of long bones: retrospective and prospective analyses. J Bone Joint Surg Am. 1976;58:453-58.

17. Ferreira N, Mare PH, Marais LC. Circular external fixator applicaltion for midshaft tibial fractures: surgical technique. South African Orthopaedic Journal. 2012;11(4):39-42.

18. Ferreira N, Marais LC. Closed distraction and gradual deformity correction of stiff hypertrophic tibial nonunions with hexapod external fixation. JBJS Essential Surgical Technique. 2016;6:e36.

19. - - - Prevention and management of external fixator pin track sepsis. Srat Traum Limb Recon. 2012;7:67-72.

20. Checketts RG, Otterburn M, MacEachern AG. Pin track infection: definition, incidence and prevention. Journal of Orthopaedic Trauma. 1993;3(Suppl):16-18

21. Weinberg DS, Park PJ, Liu RW. Association between tibial malunion deformity parameters and degenerative hip and knee disease. J Orthop Trauma. 2016;30:510-15.

22. Ferreira N, Marais LC, Aldous C, Management of tibial non-unions: prospective evaluation of comprehensive treatment algorithm. South African Orthopaedic Journal. 2016;15(1).

23. Zuidema W, Breedveld P. Two level (segmental) tibia fracures, management and complications. The Internet Journal of Orthopaedic Surgery. 2006;4.

24. Mcmurtry J, Mounasamy V. Segmental tibia fractures. Annals of Orthopedics and Rheumatology. 2015;3:1051.

25. McMahon SE, Little ZE, Smith TO, Trompeter A, aHing CB. The management of segmental tibial shaft fractures: a systematic review. Injury. 2016;47:568-73.

26. Pavolini B, Maritato M, Turelli L, D’Arienzo M. The Ilizarov fixator in trauma: a 10-year experience. J Orthop Sci. 2000;5:108-13.

27. Kummer FJ. Biomechanics of the Ilizarov external fixator. Bull Hosp Jt Dis Orthop Inst. 1989;49:140-47.

28. Ilizarov GA, ed. The apparatus: components and biomechanical principles of application. ed. by S Green, Transosseus Osteosynthesis. Theoretical and Clinical Aspects of the Regeneratin and Growth of Tissue. (Berlin: Springer-Verlag, 1992), pp. 63-136.

29. Ilizarov GA. Clinical application of the tension-stress effect for limb lengthening. Clin Orthop Relat Res. 1990;250:8-26.

30. Schwartsman V, Martin SN, Ronquist RA, Schwartsman R. Tibial fractures. The llizarov alternative. Clin Orthop Relat Res. 1992;278:207-16. 